\title{
Electrical characterization of in situ polymerized polyaniline thin films
}

\author{
S.A. Travain ${ }^{\text {a }}$, G.F. Leal Ferreira ${ }^{\text {b }}$, J.A. Giacometti ${ }^{\text {c }}$, R.F. Bianchi ${ }^{\text {a,* }}$ \\ a Departamento de Física-ICEB, UFOP, CEP 35400-000 Ouro Preto, MG, Brazil \\ ${ }^{\mathrm{b}}$ Instituto de Física de São Carlos, USP, CEP 13560-970 São Carlos, SP, Brazil \\ ${ }^{c}$ Faculdade de Ciências e Tecnologia, UNESP, CEP 19060-900 Presidente Prudente, SP, Brazil
}

Received 7 March 2007; received in revised form 18 July 2007; accepted 21 July 2007

\begin{abstract}
The alternating conductivity, $\sigma^{*}(f)=\sigma^{\prime}(f)+i \sigma^{\prime \prime}(f)$, of in situ polymerized polyaniline thin films doped with hydrochloric acid, deposited on top of an interdigitated gold line array previously deposited on glass substrates, were measured in the frequency $(f)$ range between $0.1 \mathrm{~Hz}$ to $10 \mathrm{MHz}$ and in the temperature range from 100 to $430 \mathrm{~K}$. The results for $\sigma^{\prime}(f)$ are typical of a disordered solid material: for frequencies lower than a certain hopping frequency $\gamma_{\text {hop }}, \log \left[\sigma^{\prime}(f)\right]$ is frequency-independent rising almost linearly for in $\log f>\gamma_{\text {hop }}$. A master curve was thus obtained by plotting the real component of the conductivity using normalized scales $\sigma^{\prime}(f) / \sigma_{\mathrm{dc}}$ and $f / \gamma_{\text {hop }}$, which is indicative of a single process operating in the whole frequency range. An expression encompassing the conduction through a disordered structure taken from previous random free energy barrier model for hopping carriers, as well a dielectric function to represent the capacitive behavior of the PAni was employed to fit the experimental results. The dielectric constant and activation energy for hopping carriers were obtained as function of the polymer doping level.
\end{abstract}

(C) 2007 Elsevier B.V. All rights reserved.

Keywords: Polyaniline; In situ polymerization; Electrical properties; Impedance spectroscopy

\section{Introduction}

Considerable attention has been given to the physical and chemical properties of conjugated polymers [1,2], mainly due to their potential applications in flexible and low-cost devices [3-5]. Among the most important and versatile materials are polyaniline (PAni) and its derivatives. PAni is frequently used in devices because of its easy preparation, good chemical stability and the possibility of behaving like an insulator, a semiconductor or a metal, through chemical doping or dedoping process [6]. Not only the understanding of the accompanying chemical processes is important [7], it is the same for the mechanism of charge transport through the polymer bulk and its correlation to the underlying polymer structure. By the in situ polymerization method [8,9], PAni films of rather good quality and controlled doping level may be produced, visually contrasting strongly with those prepared by casting [10]. Although amorphous, samples prepared by chemical in situ method (see

\footnotetext{
* Corresponding author.

E-mail address: bianchi@iceb.ufop.br (R.F. Bianchi).
}

Section 2) are thinner, of the order of a hundred nanometers, and much more regular than those obtained by casting. Indeed, the interpretation of conductivity measurements in cast samples [10] required the assumption of a non-homogeneous medium, since the doping in the polymer bulk is not uniform $[10,11]$. For comparison, dc and ac conductivity measurements were carried out in in situ samples, under varied doping levels and different temperatures. We have observed that doping makes samples to go from a conductive dielectric behavior to an almost metallic one. A broad temperature variation of the (real) conductivity was observed in samples with a somewhat intermediate doping level. In all cases in which the frequency dependent behavior became manifest, we were able to fit the real part of the conductivity using an expression derived from the random free energy barrier (RFEB) model [12], clearly defining a 'hopping frequency' and leading to a master curve indicative of universality. This shows that a single process would be involved in the whole frequency range and not as thick PAni films prepared by casting [10] and other amorphous materials, such as chalcogenides, in which clusters contribute exclusively to the ac conduction [13]. Concerning the imaginary component, we find a dielectric response superimposed on 
the conductivity component at low doping levels, but not for high levels.

\section{Experimental procedure}

Highly doped in situ polymerized polyaniline (PAni) thin films were obtained onto interdigitated chromium-gold line arrays deposited on glass. The PAni film was around $180 \mathrm{~nm}$ thick, covering an area of $3.3 \times 10^{-8} \mathrm{~m}^{2}$, and the gold fingers - which served as electrodes [14] - were $400 \mu \mathrm{m}$ apart. The in situ method to produce the PAni film is described elsewhere [10], while the method used to measure and control de polymer thickness was based on Ref. [15]. In order to investigate the chemical doping level of the polymer films, samples were undoped in $1 \mathrm{M} \mathrm{NH}_{4} \mathrm{OH}$ aqueous solution for $15 \mathrm{~min}$, and then doped by dipping into aqueous solutions with $\mathrm{HCl}$ concentrations varying from $10^{-4}$ to $1 \mathrm{M}$, for $15 \mathrm{~min}$. Since PAni is not completely undoped during its immersion in the $1 \mathrm{M} \mathrm{NH}_{4} \mathrm{OH}$ solution [10], the resulting sample is weakly doped. The films doped in $10^{-3} \mathrm{M} \mathrm{HCl}$ and in $10^{-2} \mathrm{M} \mathrm{HCl}$ will be referred to as moderately and intermediately doped ones, respectively. Visually, low-doped samples have a dark blue color (emeraldine base) while the highly doped are bright green (emeraldine salt).

Current versus voltage measurements ( $I$ versus $V$ ) were carried out in the range between 0 and $6 \mathrm{~V}$ with a 237 Keithley measuring unit. The ac measurements were performed using a 1260 Solartron Frequency-Response Analyzer in the $0.1-10^{7} \mathrm{~Hz}$ frequency range, voltage amplitude equal to $50 \mathrm{mV}$ (maximum ac electric field of the order of $10^{3} \mathrm{~V} / \mathrm{m}$ ), and bias voltage equal to zero. All measurements were carried out using the interdigitated gold line electrodes structure described in Ref. [14], and using an APD cryostat with temperatures varying from 100 to $430 \mathrm{~K}$, in the dark to avoid photocurrents and in a vacuum of $10^{-2}$ Torr.

We employ the following nomenclature for the sample doping level. For weak doping, the samples prepared with $10^{-4} \mathrm{M}$ $\mathrm{HCl}$ will be referred to as $W-10^{-4}$. The samples with moderate doping - with $10^{-3} \mathrm{M} \mathrm{HCl}$ - will be $M-10^{-3}$; those with intermediate doping - prepared with $10^{-2} \mathrm{M} \mathrm{HCl}$ - will be referred to as $I-10^{-2}$ and the sample with highest doping, prepared with $1 \mathrm{M} \mathrm{HCl}$, will be $H-1$. Measurements with intermediate doping levels were also performed, but to avoid repetition only the figures will be mentioned.

\section{Analysis of the alternating conductivity}

In the Introduction, we mentioned that our results support a single transport process operating in the whole frequency range. To our knowledge, only the random free energy barrier (RFEB) model of Dyre [12] covers both the low and high frequency regimes, as well as the real and imaginary components of complex conductivity, $\sigma^{*}(\omega)$, which then prompted us to apply it to our data. Dyre's expression for the disordered $\sigma^{*}(\omega)=\sigma^{\prime}(\omega)+i \sigma^{\prime \prime}(\omega)$, with $\omega=2 \pi f$, [10] is

$\sigma^{*}(\omega)=\sigma_{0}\left[\frac{i \omega / \gamma_{\text {hop }}}{\ln \left(1+i \omega / \gamma_{\text {hop }}\right)}\right]$
$\sigma_{0}=C \gamma_{\text {hop }}$

in which $\gamma_{\text {hop }}$ will be referred to here as the hopping frequency (it is the hopping frequency corresponding to the largest energy barrier in [12]), $\sigma_{0}$ the low-frequency or dc conductivity $\left(\sigma_{\mathrm{dc}}\right)$, $C$ a constant [12] and $i$ is the imaginary unit. The real part of $\sigma^{*}$ gives the constant $\sigma_{0}$ for $\omega \ll \gamma_{\text {hop }}$ and increases sub-linearly for $\omega>\gamma_{\text {hop }}$, while the imaginary part steadily rises slightly sublinearly in the whole frequency range. It can be shown from Eq. (1) a master curve may be obtained in the $\sigma^{\prime} / \sigma_{0}$ versus $\omega / \gamma_{\text {hop }}$ plot, and this universality is stronger than the Taylor-Isard sealing defined in [16]. The expected dependence on the temperature and doping is carried by $\gamma_{\text {hop }}$, according to the expression:

$\gamma_{\text {hop }}=v_{0} \mathrm{e}^{-2 \alpha r_{0}} \mathrm{e}^{-W / k T}$

where $v_{0}$ is the escape frequency, $\alpha$ the overlap parameter, $r_{0}$ the mean hopping distance, $W$ an activation energy, $T$ the absolute temperature and $k$ is the Boltzmann constant. Of course, $r_{0}$ depends on the doping, expected to vary as the inverse of the cubic root of the dopant concentration $c_{\text {bulk }}$ [17]:

$r_{0} \propto c_{\mathrm{bulk}}^{-1 / 3}$

As we shall show, our data point to a capacitive behavior for samples with low doping level, and therefore the total conductivity $\sigma_{\text {tot }}^{*}(w)$ is [18]

$\sigma_{\text {tot }}^{*}(\omega)=\sigma^{*}(\omega)+i \omega \varepsilon \varepsilon_{0}$

where $\varepsilon$ is the dielectric constant and $\varepsilon_{0}$ is the vacuum permittivity. However, for samples with higher doping level no such contribution seems to be present.

\section{Experimental results and fitting}

Fig. 1 shows $I$ versus $V$ curves obtained with the samples $W-10^{-4}$ (Fig. 1a), $M-10^{-3}$ (Fig. 1b), $I-10^{-2}$ (Fig. 1c) and $H-1$ (Fig. 1d), displaying Ohmic behavior in all cases. The dc conductivity $\left(\sigma_{\mathrm{dc}}\right)$ increases with the acid concentration, being equal to $1.8 \times 10^{-9}, 3.3 \times 10^{-5}, 6.5 \times 10^{-3}$ and $1.6 \times 10^{-1} \mathrm{~S} / \mathrm{m}$ for $W-10^{-4}, M-10^{-3}, I-10^{-2}$ and $H-1$ samples, respectively. This rather broad linear behavior range is related to the matching of the Au work function with the PAni ionization potential, both around to $5.4 \mathrm{eV}$, thus leading to a neutral contact $[17,19]$. Thus, the bulk conduction is then governed by the transport of holes in the polymer bulk.

Fig. 2 shows the real part of the conductivity, $\sigma^{\prime}(f)$, obtained for different doping levels at $300 \mathrm{~K}$, including the same set of PAni films shown in Fig. 1. From weakly to moderately doped samples (curves a to c), $\log \left[\sigma^{\prime}(f)\right]$ is frequency independent at low frequencies. It increases above a critical frequency, $\gamma_{\text {hop }}$, which is the hopping frequency in our previous equations. As expected, $\sigma^{\prime}(f \rightarrow 0)$, or $\sigma_{\mathrm{dc}}$, agrees with those in Fig. 1. Finally, it may be inferred that strongly doped samples (curves $d$ to g) behave like a metal, in the sense that its real component is frequency independent in the whole frequency range.

The effect of the temperature on $\sigma^{\prime}(f)$ is displayed in Fig. 3 for measurements carried out in $W-10^{-4}$ (Fig. 3a), $M-10^{-3}$ 

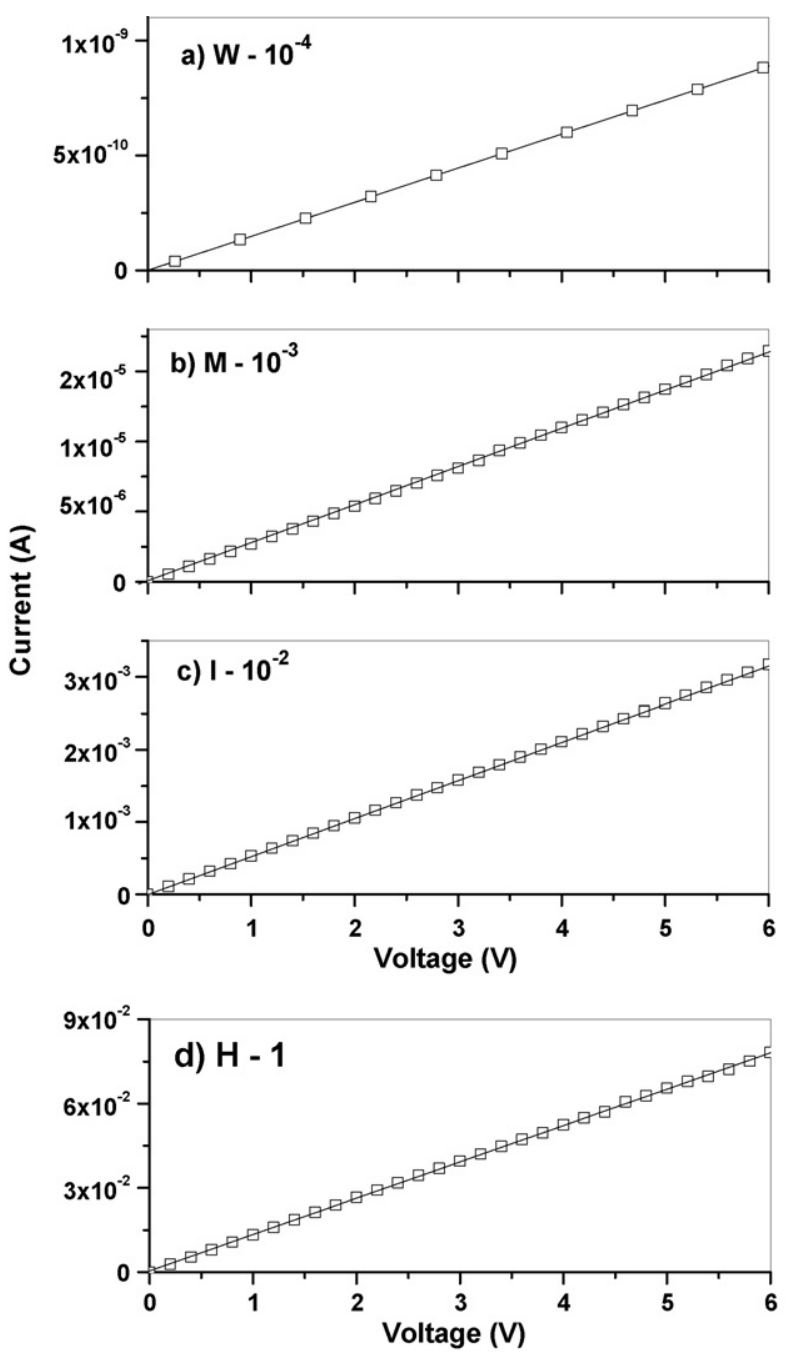

Fig. 1. Current vs. voltage measurements on samples: (a) $W-10^{-4}$, (b) $M-10^{-3}$, (c) $I-10^{-2} \mathrm{e}$ and (d) $H-1 \mathrm{HCl}$ doped PAni films. Measurements were carried out at $300 \mathrm{~K}$ and under vacuum.

(Fig. 3b) and $I-10^{-2}$ (Fig. 3c) samples. For the $W-10^{-4}$ sample the temperature was varied from 300 to $430 \mathrm{~K}$. As expected, an increase in temperature causes the conductivity to increase, as the doping does (Fig. 2), but the metallic behavior is reached for the $I-10^{-2}$ doped sample (Fig. 3c) only at temperatures as high as $250 \mathrm{~K}$. Significantly, a master curve can be obtained from the data in Figs. 2 and 3 using the normalization $\sigma^{\prime}(f) / \sigma_{0}$ and $f / \gamma_{\text {hop. }}$. Such universality, illustrated in Fig. 4, was also observed for the other disordered materials [16].

For the sake of completeness $\sigma^{\prime}(f)$ and $\sigma^{\prime \prime}(f)$ are shown for the three lower doping levels, respectively, in Fig. 5a, b and c at $300 \mathrm{~K} . \sigma^{\prime \prime}(f)$ is practically independent of the polymer doping level: considering the extreme values, it is close to $10^{-10} \mathrm{~S} / \mathrm{m}$ for $f=0.1 \mathrm{~Hz}$, and $10^{-3} \mathrm{~S} / \mathrm{m}$ for $f=10 \mathrm{MHz}$. This indicates that $\sigma^{\prime \prime}(f)$ derives mainly from a capacitive component in parallel with the conduction process [18] (see Eq. (5)). However, for the $I-10^{-2}$ sample, $\sigma^{\prime \prime}(f)$ seems to derive entirely from the conduction contributions, as will be discussed in the next section.

The temperature dependence is illustrated in Fig. 6, which shows $\sigma^{\prime}(f)$ and $\sigma^{\prime \prime}(f)$ versus $f$ for the moderately doped

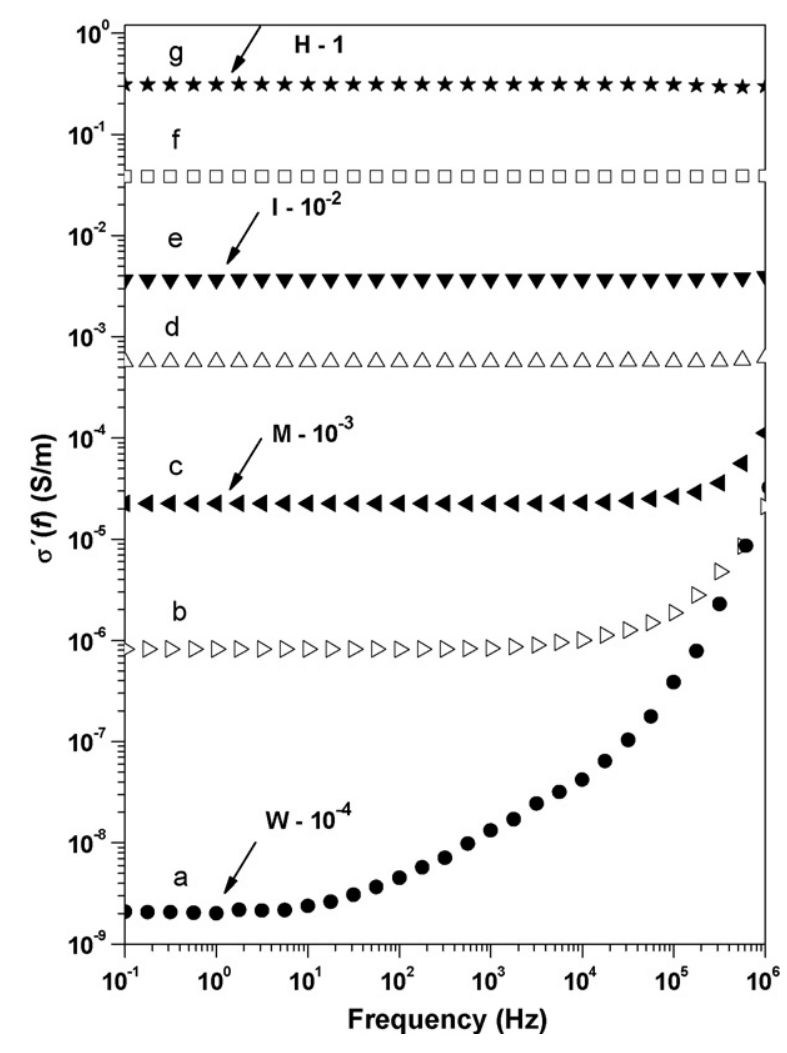

Fig. 2. Real component, $\sigma^{\prime}(f)$, of the ac vs. frequency of in situ polymerized PAni thin films doped with different $\mathrm{HCl}$ aqueous solutions, as indicated by the curves (a) $10^{-4} \mathrm{M}\left(W-10^{-4}\right)$, (b) $5 \times 10^{-4} \mathrm{M}$, (c) $10^{-3} \mathrm{M}\left(M-10^{-3}\right)$, (d) $5 \times 10^{-3} \mathrm{M}$, (e) $10^{-2} \mathrm{M}\left(I-10^{-2}\right)$, (f) $10^{-1} \mathrm{M}$, and (g) $1 \mathrm{M}(H-1)$.

$M-10^{-3}$ sample, at three temperatures. The results are similar to those shown in Fig. 2 for different doping levels: $\sigma^{\prime}(f \rightarrow 0)$ and $\gamma_{\text {hop }}$ increase with the polymer doping level and temperature, while $\sigma^{\prime \prime}(f)$ remains unchanged. Full lines in Figs. 5 and 6 represent the theoretical fittings with the model from Section 3, in which $\sigma_{0}$ estimated from the dc measurements was used as input parameter. It is important to remark that the linear ranges of $I$ versus $V$ curves are compressed at lower temperature. This means that the value of dc conductivity at low temperature will change, if temperature changes in agreement with Ref. [21]. Finally, the model was used to fit the data for the other samples using Eqs. (1), (2) and (5), and the parameters employed are summarized in Table $1 \mathrm{sdc}$ was obtained from dc measurements, Fig.1.

\section{Discussion}

In Dyre's model $[12,16]$, the quantity actually calculated is a jumping frequency, with the conductivity being taken as proportional to this frequency with a proportionality factor $C$ in Eq. (2). Though this assumption has become commonplace (see [17]), it is still unclear how $C$ depends on the doping level. We infer from Table 1 that despite the large changes in doping and conductivity, it is remarkable that $C=\sigma_{0} / \gamma_{\text {hop }}$ remains practically a constant, of the order of $5 \times 10^{-10} \mathrm{~F} / \mathrm{m}$, with only a weak dependence on the temperature. For the $I-10^{-2}$ sample, it is somewhat higher, $10^{-9} \mathrm{~F} / \mathrm{m}$. These results show that the 

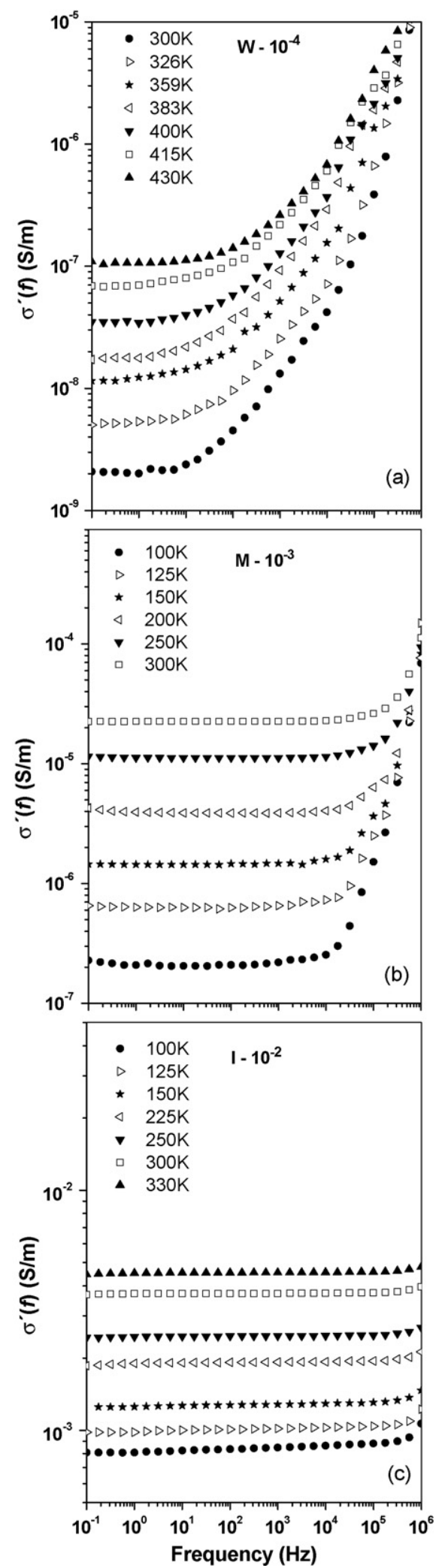

Fig. 3. Real component, $\sigma^{\prime}(f)$, at different temperatures, from 100 to $430 \mathrm{~K}$ : (a) for $W-10^{-4}$, (b) $M-10^{-3}$, and (c) $I-10^{-2}$.

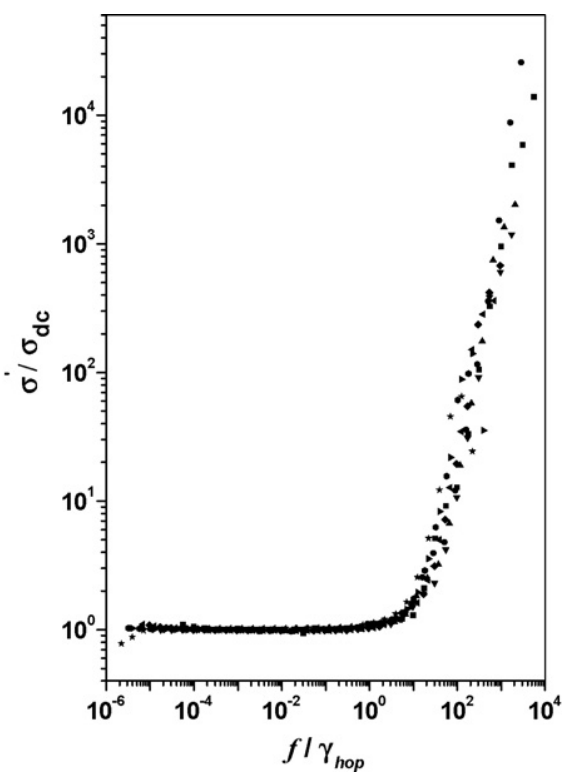

Fig. 4. Master curve obtained from results of Fig. 2(a, b, and c) and also from all curves of Fig. 3b. These results obtained were normalized: $\sigma^{\prime}(f)$ divided by $\sigma_{\mathrm{dc}}$ and $f$ divided by $\gamma_{\text {hop }}$.

Table 1

Adjusted parameters obtained from the fittings according to Eqs. (1), (2) and (5) for $W-10^{-4}, M-10^{-3}$, and $I-10^{-2}$ PAni samples

\begin{tabular}{|c|c|c|c|c|c|}
\hline $\begin{array}{l}\text { Temperature } \\
(\mathrm{K})\end{array}$ & $\begin{array}{l}\sigma_{\mathrm{dc}} \\
\left(\times 10^{-9} \mathrm{~S} / \mathrm{m}\right)\end{array}$ & $\begin{array}{l}\sigma_{0} \\
\left(\times 10^{-9} \mathrm{~S} / \mathrm{m}\right)\end{array}$ & $\begin{array}{l}\gamma_{\text {hop }} \\
(\mathrm{Hz})\end{array}$ & $\begin{array}{l}C=\sigma_{0} / \gamma_{\text {hop }} \\
10^{-10}\end{array}$ & $\varepsilon$ \\
\hline \multicolumn{6}{|c|}{$10^{-4} \mathrm{M}$ (weakly doped) } \\
\hline 300 & 2.5 & 2.5 & 8 & 3.1 & 18 \\
\hline 315 & 3.6 & 3.5 & 11 & 3.2 & 18 \\
\hline 326 & 5.2 & 5.3 & 14 & 3.8 & 18 \\
\hline 337 & 7.4 & 7.4 & 16 & 4.6 & 18 \\
\hline 345 & 8.5 & 9.5 & 18 & 5.2 & 18 \\
\hline 359 & 11.8 & 12.0 & 20 & 6.0 & 18 \\
\hline 371 & 12.4 & 13.5 & 21 & 6.2 & 18 \\
\hline 383 & 16.5 & 18.5 & 22 & 8.2 & 18 \\
\hline 393 & 18.3 & 22.5 & 22 & 10.2 & 18 \\
\hline $\begin{array}{l}\text { Temperature } \\
(\mathrm{K})\end{array}$ & $\begin{array}{l}\sigma_{\mathrm{dc}} \\
\left(\times 10^{-7} \mathrm{~S} / \mathrm{m}\right)\end{array}$ & $\begin{array}{l}\sigma_{0} \\
\left(\times 10^{-7} \mathrm{~S} / \mathrm{m}\right)\end{array}$ & $\begin{array}{l}\gamma_{\text {hop }} \\
\left(\times 10^{3} \mathrm{~Hz}\right)\end{array}$ & $\begin{array}{l}C=\sigma_{0} / \gamma_{\text {hop }} \\
10^{-10}\end{array}$ & $\varepsilon$ \\
\hline \multicolumn{6}{|c|}{$10^{-3} \mathrm{M}$ (moderately doped) } \\
\hline 100 & 2.1 & 2 & 2 & 0.9 & 15 \\
\hline 125 & 6.4 & 6 & 3 & 1.7 & 15 \\
\hline 150 & 14.4 & 15 & 5 & 2.7 & 15 \\
\hline 175 & 27.1 & 26 & 7 & 3.5 & 15 \\
\hline 200 & 39.3 & 37 & 12 & 3.0 & 15 \\
\hline 225 & 63.1 & 60 & 17 & 3.5 & 15 \\
\hline 250 & 113.0 & 110 & 28 & 3.8 & 15 \\
\hline 275 & 162.0 & 160 & 34 & 4.7 & 15 \\
\hline 300 & 219.0 & 230 & 48 & 4.8 & 15 \\
\hline $\begin{array}{l}\text { Temperature } \\
\text { (K) }\end{array}$ & $\begin{array}{l}\sigma_{\mathrm{dc}} \\
\left(\times 10^{-3} \mathrm{~S} / \mathrm{m}\right)\end{array}$ & $\begin{array}{l}\sigma_{0} \\
\left(\times 10^{-3} \mathrm{~S} / \mathrm{m}\right)\end{array}$ & $\begin{array}{l}\gamma_{\text {hop }} \\
\left(\times 10^{5} \mathrm{~Hz}\right)\end{array}$ & $\begin{array}{l}C=\sigma_{0} / \gamma_{\text {hop }} \\
10^{-9}\end{array}$ & $\varepsilon$ \\
\hline \multicolumn{6}{|c|}{$10^{-2} \mathrm{M}$ (intermediate doped) } \\
\hline 175 & 1.1 & 1.1 & 4 & 2.8 & - \\
\hline 200 & 1.5 & 1.5 & 4 & 3.3 & - \\
\hline 225 & 1.9 & 1.9 & 5 & 3.8 & - \\
\hline 250 & 2.5 & 2.5 & 7 & 3.5 & - \\
\hline 275 & 3.2 & 3.2 & 9 & 3.3 & - \\
\hline 300 & 3.8 & 3.7 & 10 & 3.7 & - \\
\hline 330 & 4.6 & 4.4 & 15 & 2.9 & - \\
\hline
\end{tabular}



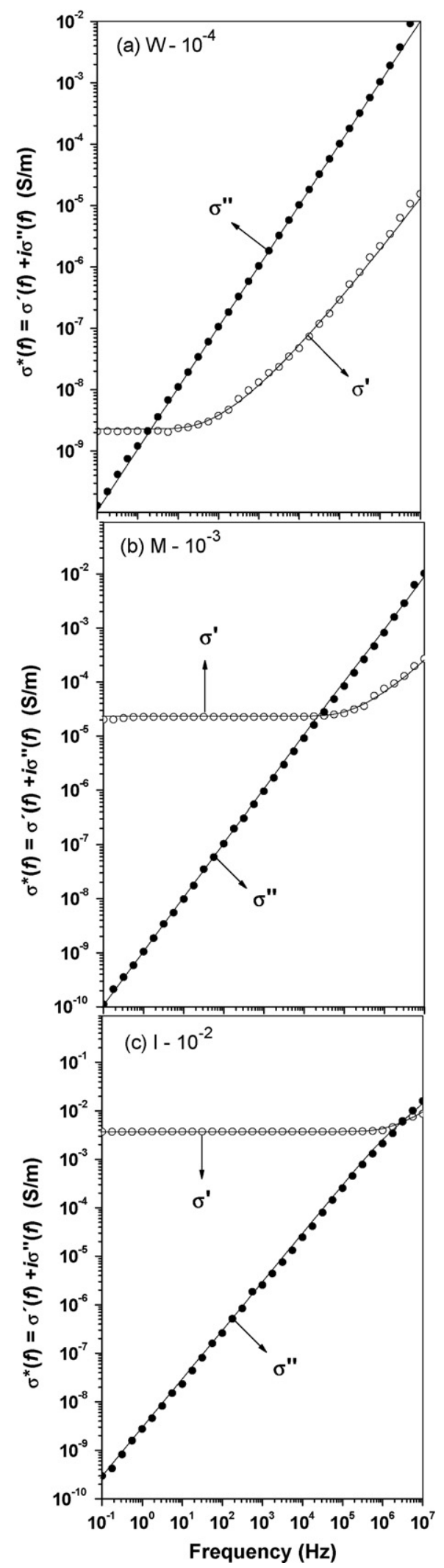

Fig. 5. Real and imaginary component of the complex conductivity vs. frequency at $300 \mathrm{~K}$ corresponding to (a) $W-10^{-4}$, (b) $M-10^{-3}$, and (c) $I-10^{-2}$ doped samples.
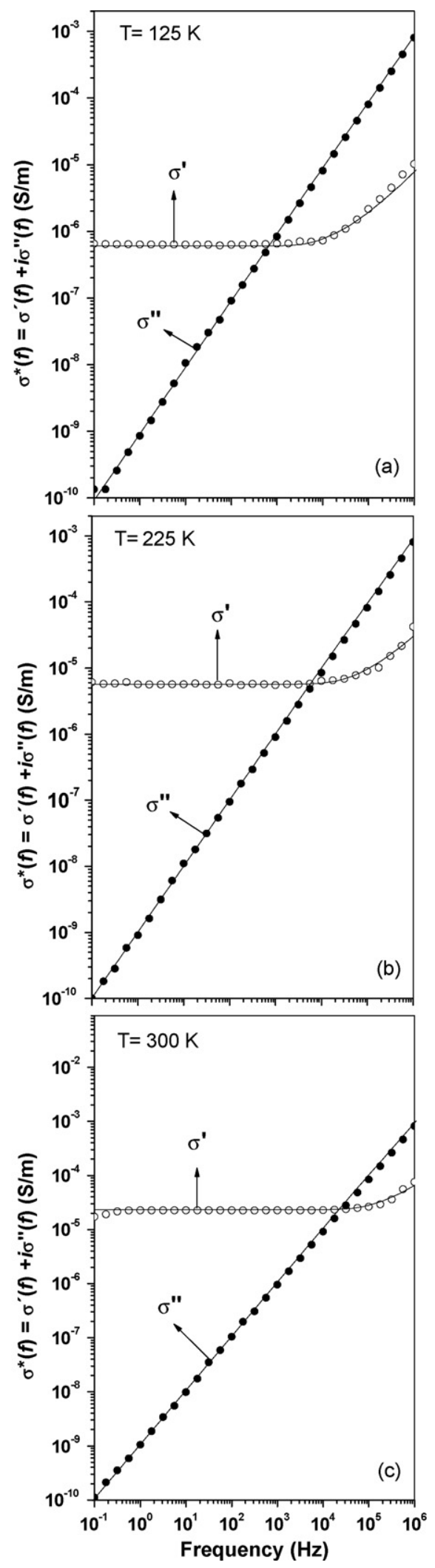

Fig. 6. Real and imaginary component of the complex conductivity of $M-10^{-3}$ samples at (a) $125 \mathrm{~K}$, (b) $225 \mathrm{~K}$, and (c) $300 \mathrm{~K}$. 


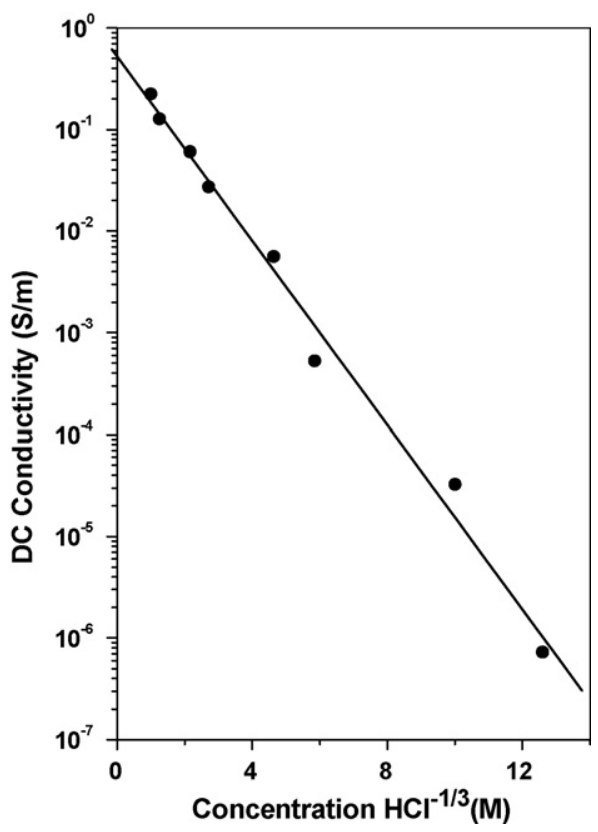

Fig. 7. $\log \sigma_{\mathrm{dc}}$ vs. $c_{\mathrm{s}}^{-1 / 3}$, at $300 \mathrm{~K} ; c_{\mathrm{s}}$ is a function of $\mathrm{HCl}$ concentration in aqueous solution employed for doping level.

dc conductivity is around $\sigma_{0}$ and thus both $\sigma_{0}$ and $\gamma_{\text {hop }}$ increase with temperature and doping level, as expected for semiconducting polymers materials $[1,10]$. On the other hand, the dielectric constant $(\varepsilon)$ is around 15-18 for weakly and moderated doped samples, and does not influence the ac conductivity behavior for high conductivity samples.

In order to test the dependence of the conductivity $\sigma_{0}$ on the doping level, the results of Fig. 2 were used, assuming additionally the bulk concentration $c_{\text {bulk }}$ to be proportional to the corresponding acid concentration $c_{\mathrm{s}}$ employed in protonation.

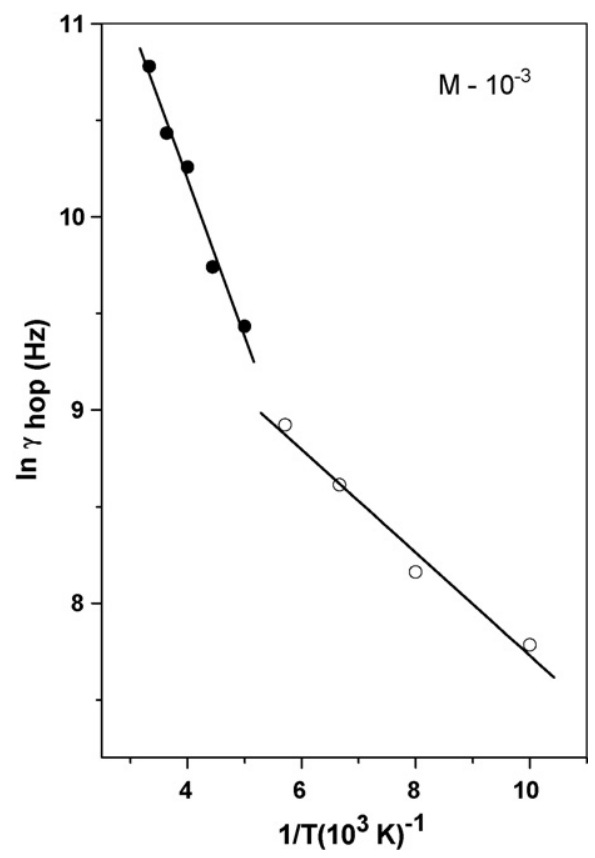

Fig. 8. Attempt to obtain the activation energy from an Arrhenius plot according to Eq. (3) for $M-10^{-3}$ sample: two activation energies result.

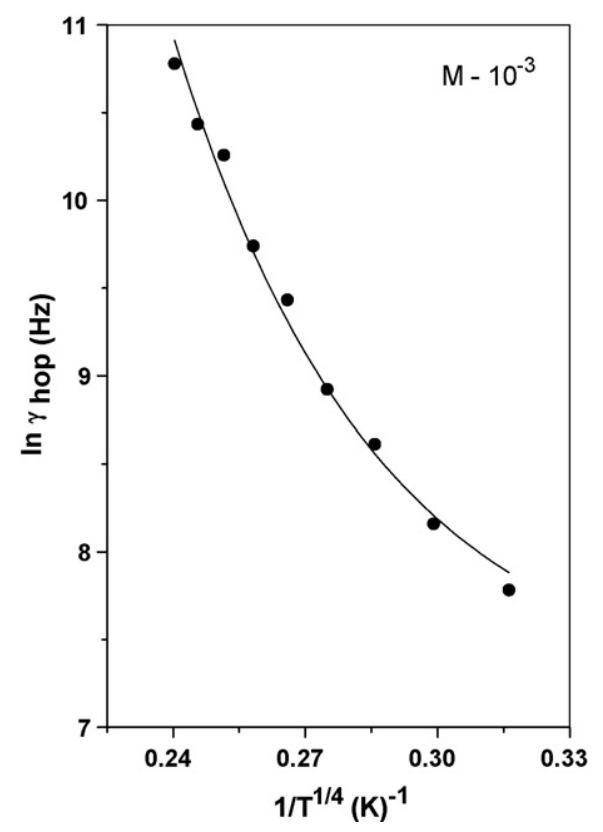

Fig. 9. $\ln \gamma_{\text {hop }}$ vs. $1 / T^{1 / 4}$. Mott's variable range hopping plot corresponding to Fig. 8: a single straight line does not result.

In Fig. 7 we plot $\sigma_{0}$ at $300 \mathrm{~K}$ as function of $c_{\mathrm{s}}^{-1 / 3}$ and we find that an approximate straight line indeed results.

Concerning the temperature dependence, only the data in Table 1 referring to $M-10^{-3}$ doping will be considered in detail. Following the suggestion in Eq. (3), a plot of $\log \left(\gamma_{\text {hop }}\right)$ versus $1 / T$ is shown in Fig. 8 and, surprisingly, two activation energies would be inferred, a lower one $W=23 \mathrm{meV}$, operating at low temperatures $T<227 \mathrm{~K}$ and a higher one $W=70 \mathrm{meV}$ at temperatures higher than $227 \mathrm{~K}$, where the temperature $\sim 227 \mathrm{~K}$ may be attributed to glass transition of the material [20]. Moreover, it may be argued that the literature offers other possible temperature dependences, for instance the famous $T^{-1 / 4}$ for the variable range hopping at low temperatures $(T<200 \mathrm{~K})$. It may be explained by the fact that the dc conductivity of polyaniline is not dependent only on the temperature and doping level, but also on the material processing and structure [20]. Furthermore, although expected to hold at low temperatures, we show in Fig. 9 the plot of $\log \left(\gamma_{\mathrm{hop}}\right)$ versus $1 / T^{1 / 4}$ and observe that the knee is indeed somewhat smoothed out, but the result cannot be said to be a straight line. For the other two doping levels, $W-10^{-4}$ and $I-10^{-2}$, the temperature range employed was narrower but even so not a single activation energy could be found, as obtained in casting films [10]. We also mentioned that a study carried out in Ref. [10,17] for casting samples also showed more complicated temperature and doping dependence.

Finally, the adjusted dielectric constant in Table 1 is analyzed. For lower doping levels $\left(10^{-4}\right.$ and $\left.10^{-3} \mathrm{M}\right)$ its contribution is higher than that from $\sigma^{\prime \prime}(f)$ in Eq. (1), and therefore Eq. (5) was employed, leading to a rather good linear $\omega$ dependence (see Figs. 5 and 6). However, for the $I-10^{-2} \mathrm{M}$, a sample already showing metallic shine (see end of Section 2), $\sigma^{\prime \prime}(f)$ in Eq. (1) gives the whole contribution and $\varepsilon$ is null. This means that even the vacuum contribution is being cancelled by the conduction mechanisms. 


\section{Final remarks}

Dyre's Eq.(1) supplemented by a doping level dependent contribution, Eq.(5), were used to explain our results in in situ doped polyaniline thin films. The increase in conductivity was explained by the increase of the hoping sites afforded by doping. As results, it is obtained not only the dielectric constant as function of doping level, but also the activation energy for hopping carriers below and above the possible glass transition of the material around $227 \mathrm{~K}$.

\section{Acknowledgments}

This work was sponsored by FAPEMIG, CNPq and IMMP/CNPq from Brazil.

\section{References}

[1] M. Pope, C.E. Swenberg, Electronic Process in Organic Crystals and Polymers, 2nd ed., Oxford University Press, Oxford, 1999.

[2] P.F. Hadziioannou, van Hutten, Semiconducting Polymers: Chemistry, Physics and Engineering, Wiley-VCH, Weinheim, 1999.

[3] J. Shinar, Organic Light-Emitting Devices, Springer, 2004.

[4] J. Kalinowski, Organic Light-Emitting Diodes, Marcel Dekker, 2005.

[5] C.J. Brabec, V. Dyakonov, J. Parisi, N.S. Sariciftci, Organic Photovoltaics: Concepts and Realization, Springer, 2003.
[6] A.G. MacDiarmid, Ang. Chem. Int. Ed. 40 (14) (2001) 2581-2590.

[7] W.R. Salaneck, S. Stagström, J.-L. Brèdas, Conjugated Polymer Surfaces and Interfaces, Cambridge University Press, 2003.

[8] A.G. MacDiarmid, A.J. Epstein, Faraday Discuss. Chem. Soc. 88 (1989) 317-332.

[9] I. Sapurina, I. Riede, J. Stejskal, Synth. Met. 123 (2001) 503-507.

[10] R.F. Bianchi, G.F.L. Ferreira, C.M. Lepienski, R.M. Faria, J. Chem. Phys. 110 (9) (1999) 4602-4607.

[11] D. Jeon, Science 256 (1992) 1662-1664.

[12] J.C. Dyre, J. Appl. Phys. 64 (1988) 2456-2468.

[13] N.F. Mott, Conduction in Non-Crystalline Materials, Oxford Clarendon Press, 1987.

[14] R.F. Bianchi, R.K. Onmori, R.M. Faria, J. Polym. Sci. B: Polym. Phys. 43 (2005) 74-78.

[15] J.L. Forveille, L. Olmedo, Synth. Met. 65 (1994) 5-11.

[16] J.C. Dyre, T.B. Schroeder, Rev. Modern Phys. 72 (2000) 873-892.

[17] R.F. Bianchi, H.N. Cunha, R.M. Faria, G.F.L. Ferreira, J.M.G. Neto, J. Phys. D: Appl. Phys. 38 (2005) 1437-1443.

[18] R.J. Ramos, R.F. Bianchi, D.T. Balogh, R.M. Faria, IEEE Trans. Dielectr. Electr. Insul. 7 (2000) 855-859.

[19] P. Barta, Th. Kugler, W.R. Salaneck, A.P. Monkman, J. Libert, R. Lazzaroni, J.L. Brèdas, Synth. Met. 93 (1998) 83-87.

[20] P. Rannou, B. Dufour, J.P. Travers, A. Pron, D. Djurado, H. Janeczek, D. Sek, J. Phys. Chem. B 106 (2002) 10553-10559.

[21] Y. Long, L. Zhang, Z. Chen, K. Huang, Y. Yang, H. Xiao, M. Wan, A. Jin, C. Gu, Phys. Rev. B 71 (2005) 1-7, 165412. 